\title{
Xylazine abuse: A rare cause of syncope
}

Keywords Xylazine; Orthostatic hypotension; Syncope

\section{To the Editor:}

Syncope is a common presentation in the emergency department. A variety of drugs should be considered as potential causes of orthostatic hypotension complicating syncope (1). We present a case of inhaled xylazine abuse with intermittent syncope, in which recovery was complete following supportive care. This case highlights the importance to the emergency physician of considering abused substances in the initial diagnosis of the causes of syncope.

\section{Case report}

A 19-year-old man with a history of chronic use of antacids and sulpiride for chronic epigastric pain presented to our emergency department after four episodes of syncope with spontaneous recovery after a few seconds. He denied any systemic disease. The syncope was preceded by symptoms of dizziness and blurred vision that improved upon lying down.

On arrival, the patient's blood pressure was $120 / 87 \mathrm{~mm}$ $\mathrm{Hg}$, heart rate was 78 beats/minute, temperature was $35.5^{\circ} \mathrm{C}$, and respiratory rate was 16 breaths/minute. Physical and neurological examinations were unremarkable and he was lucid. A complete ECG revealed sinus bradycardia. Routine hematology and biochemistry values were all within normal limits. However, significant orthostatic hypotension was confirmed according to the consensus of the American Autonomic Society (AAS) and the American Academy of Neurology (AAN; see Table 1). The patient was admitted to the neurology ward for possible autonomic nervous system (ANS) dysfunction. However, echocardiography, 24-hour Holter ECG, nerve conduction velocity, sympathetic skin response, and RR interval variability revealed no positive findings. A urine drug screening (REMEDi HS, Bio-Rad Laboratories, Hercules, CA) revealed the presence of xylazine and sulpiride. Therefore,

Received 8 February 2006; accepted 10 February 2006.

This study was supported by the Ta-Tung Kidney Foundation.

Address correspondence to Cheng-Chung Fang, M.D., Department of Emergency Medicine, National Taiwan University Hospital, College of Medicine, National Taiwan University, No. 7, Chung-Shan South Road, Taipei 100, Taiwan, R.O.C. E-mail: conrad@ha.mc.ntu.edu.tw we screened the urine sample with gas chromatography/mass spectrometry (GC/MS, Agilent 5890 gas chromatograph coupled with a 5973 mass selective detector), and found the presence of ketamine, norketamine, phenobarbital, and xylazine. The concentrations of these compounds were confirmed by GC/MS as $582 \mathrm{mg} / \mathrm{L}$ of ketamine, $448 \mathrm{mg} / \mathrm{L}$ of norketamine, $745 \mathrm{mg} / \mathrm{L}$ of phenobarbital, and $762 \mathrm{mg} / \mathrm{L}$ of xylazine. The patient admitted to drug abuse over the previous six months. He inhaled unknown powders and began to suffer from repeated episodes of syncope. After supportive treatment (including intravenous $0.9 \%$ saline at a rate of 2000 $\mathrm{mL}$ per day), no additional episodes of orthostatic hypotension were identified beyond day four of hospitalization (Table 1). The patient was discharged without any sequelae.

\section{Discussion}

Xylazine is used as a non-narcotic sedative used in veterinary medicine $(2,3)$. Xylazine, like clonidine, stimulates presynaptic $\alpha_{2}$-adrenergic receptors resulting in a decreased release of adrenaline from nerve terminals $(4,5)$. Neurological effects include ataxia, hypotonia, lethargy, coma, respiratory depression, and apnea (6). Cardiovascular effects include an initial hypertension with sustained hypotension and increased vagal tone-related bradyarrhythmogenic effects predominating (3). Because of the pronounced hypotensive effects of xylazine in humans, xylazine is restricted for use as a veterinary medicine (5).

Most cases of xylazine intoxication are intentional. The routes of administration include intravenous, intramuscular, oral, and inhalation. The most common presentations of xylazine intoxication include consciousness disturbance potentially requiring ventilator support, bradycardia, and hypotension. Miosis and hyperglycemia have also been noted $(6,7)$. Only two cases of intentional xylazine intoxication by inhalation have been reported previously $(6,8)$. In the first case, the patient was discovered in a coma with intermittent apnea and bradycardia. He recovered completely after hydration and ventilator support (6). In the second case, the patient inhaled xylazine several times. His presentation included disorientation, hypotension, bradycardia, dysarthria, syncope, and ataxia. He recovered completely after hydration (8). Our patient represents the third case report of inhaled xylazine intoxication. In 
Table 1. Blood pressure and heart rate changes with position change

\begin{tabular}{lcccc}
\hline Day & Reading, position & $\begin{array}{c}\text { Blood pressure } \\
(\mathrm{mmHg})\end{array}$ & $\begin{array}{c}\text { Heart rate } \\
\text { (beats/min) }\end{array}$ & Symptoms \\
\hline $1 \quad$ Baseline, supine \\
position for 30 & $120 / 87$ & 78 & No \\
minutes & & & \\
Upright, standing \\
for 1 minute \\
Baseline, supine \\
position for 30 \\
minutes \\
Upright, standing \\
for 1 minute \\
Upright, standing \\
for 3 minutes
\end{tabular}

Definition of orthostatic hypotension is according to the American Autonomic Society (AAS) and the American Academy of Neurology (AAN): systolic blood pressure decrease of at least $20 \mathrm{mmHg}$ or a diastolic blood pressure decrease of at least $10 \mathrm{mmHg}$ within 3 minutes of standing (12). The examination was terminated after standing for 1 minute because of severe dizziness and near fainting on day 1 of hospitalization. The examination was completed and revealed adequate physiological response on day 4 of hospitalization.

particular, the patient presented with orthostatic hypotensionrelated syncope without other neurological signs.

The treatment of xylazine intoxication is supportive. Endotracheal intubation may be needed in cases with significant respiratory depression. Symptomatic bradycardia may respond to atropine and fluid resuscitation should be performed for hypotension. Vasopressor agents may also be helpful (6). Because limited data are available on the use of antagonists such as yohimbine, tolazoline, and atipamizole, these agents should be reserved for use in unresponsive patients only $(4,6,8)$. Although xylazine may interact with opioid receptors, naloxone has no effect in reversing xylazine intoxication (4).

In cases of unexplained repeated syncope, drug abuse should be considered in the differential diagnosis. Drugs that can induce orthostatic hypotension are classified into several categories (1). The first category includes drugs that induce the cardiovascular system, complicating hypotension with either bradycardia or tachycardia. All antihypertensive agents, sympatholytics, monoamine oxidase inhibitors, and phosphodiesterase inhibitors are in this group. The second category includes the prolonged use of sympathomimetics such as $\beta$-adrenergic agonist, ephedrine, amphetamine, and cocaine, which can induce orthostatic hypotension, complicating tachycardia-related decreased cardiac output. The third category includes drugs that induce hypotensive effects related to severe CNS depression. All narcotics, sedatives, tranquilizers, tricyclic antidepressants, anticonvulsants, and alcohol are in this group. Xylazine may be included in this group, but it possesses the most obvious hypotensive effects even in the absence of sedation, a clue to assist emergency physician's diagnosis.
In our case, ketamine, phenobarbital, sulpiride, and xylazine were detected in the patient's urine. Although phenobarbital may cause CNS depression leading to cardiovascular collapse (10), there are no clinical reports of phenobarbital causing repeated orthostatic hypotension. Hypotensive episodes secondary to cardiac arrhythmias were reported in patients with sulpiride overdose (11), but hypertension and tachycardia are the most common effects of sulpiride (12). Ketamine stimulates the sympathetic nervous system and usually presents hypertension and tachycardia (13). One case of simultaneous intoxication with xylazine and ketamine has been reported. In that case, hypertension and sinus tachycardia were presenting features (13). In our case, although the precise duration of exposure and the definite serum levels of these drugs were unavailable, xylazine seemed to play the major role in the unusual presentation.

For emergency physicians, syncope is a common symptom that may be difficult to diagnose. In cases of syncope that present with orthostatic hypotension and bradycardia but without neurological manifestations, xylazine intoxication should be considered by the emergency physician in the differential diagnosis.

Chang-Ming Liu, M.D. Department of Emergency Medicine, Far Eastern Memorial Hospital, Taipei City, Taipei, Taiwan, R.O.C.

Ming-Jang Chiu, M.D. Department of Neurology, National Taiwan University Hospital, College of Medicine, National Taiwan University, Taipei, Taiwan, R.O.C.

Cheng-Chung Fang, M.D. Wen-Jone Chen, M.D., Ph.D. Department of Emergency Medicine, National Taiwan University Hospital, College of Medicine, National Taiwan University, Taipei, Taiwan, R.O.C.

\section{References}

1. Bradley JG, Davis KA. Orthostatic hypotension. Am Fam Physician 2003; 68:2393-2398.

2. Tranquilli WJ, Benson GJ. Advantages and guidelines for alpha-2 agonists as anesthetic adjuvants. Vet Clin North Am Small Anim Pract 1992; 22:289-293.

3. Paddleford RR, Harvey RC. Alpha-2 agonists and antagonists. Vet Clin North Am Small Anim Pract 1999; 29:737-745.

4. Hoffmann U, Meister CM, Golle K, Zschiesche M. Severe intoxication with the veterinary tranquilizer xylazine in humans. J Anal Toxicol 2001; 25:245-249.

5. Mittleman RE, Hearn WL, Hime GW. Xylazine toxicity-literature review and report of two cases. J Forensic Sci 1998; 43:400-402.

6. Capraro AJ, Wiley II JF, Tucker JR. Severe intoxication from xylazine inhalation. Pediatr Emerg Care 2001; 17:447-448. 
7. Spoerke DG, Hall AH, Grimes MJ, Honea III BN, Rumack BH. Human overdose with the veterinary tranquilizer xylazine. Am J Emerg Med 1986; 4:222-224.

8. Elejalde JI, Louis CJ, Elcuaz R, Pinillos MA. Drug abuse with inhalated xylazine. Eur J Emerg Med 2003; 10:252-253.

9. Gallanosa AGE, Spyker DA, Shipe JR. Human xylazine overdose: A comparative review with clonidine, phenothiazines, and tricyclic antidepressants. Clin Toxicol 1981; 18:663-678.
10. Barile FA. Sedative/Hypnotics. In: Clinical toxicology: Principles and mechanisms. 2003:109-20.

11. Capel MM, Colbridge MG, Henry JA. Overdose profiles of new antipsychotic agents. Int J Neuropsychopharmacol 2000; 3:51-54.

12. Rossi F, Forgione A. Pharmacotoxicological aspects of levosulpiride. Pharmacol Res 1995; 31:81-94.

13. Arican FO, Okan T, Badak O, Guneri S. An unusual presentation from xylazine-ketamine. Vet Hum Toxicol 2004; 46:324-325. 